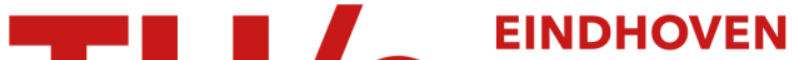 UNIVERSITY OF TECHNOLOGY
}

\section{On analytical expressions for the distribution of the filtered output of square envelope receivers with signal and colored Gaussian noise input}

\section{Citation for published version (APA):}

Tafur Monroy, I. (2001). On analytical expressions for the distribution of the filtered output of square envelope receivers with signal and colored Gaussian noise input. IEEE Transactions on Communications, 49(1), 19-23. https://doi.org/10.1109/26.898245

DOI:

$10.1109 / 26.898245$

Document status and date:

Published: 01/01/2001

\section{Document Version:}

Publisher's PDF, also known as Version of Record (includes final page, issue and volume numbers)

\section{Please check the document version of this publication:}

- A submitted manuscript is the version of the article upon submission and before peer-review. There can be important differences between the submitted version and the official published version of record. People interested in the research are advised to contact the author for the final version of the publication, or visit the DOI to the publisher's website.

- The final author version and the galley proof are versions of the publication after peer review.

- The final published version features the final layout of the paper including the volume, issue and page numbers.

Link to publication

\footnotetext{
General rights

- You may freely distribute the URL identifying the publication in the public portal. follow below link for the End User Agreement:

www.tue.nl/taverne

\section{Take down policy}

If you believe that this document breaches copyright please contact us at:

openaccess@tue.nl

providing details and we will investigate your claim.
}

Copyright and moral rights for the publications made accessible in the public portal are retained by the authors and/or other copyright owners and it is a condition of accessing publications that users recognise and abide by the legal requirements associated with these rights.

- Users may download and print one copy of any publication from the public portal for the purpose of private study or research.

- You may not further distribute the material or use it for any profit-making activity or commercial gain

If the publication is distributed under the terms of Article $25 \mathrm{fa}$ of the Dutch Copyright Act, indicated by the "Taverne" license above, please 


\title{
On Analytical Expressions for the Distribution of the Filtered Output of Square Envelope Receivers with Signal and Colored Gaussian Noise Input
}

\author{
Idelfonso Tafur Monroy
}

\begin{abstract}
Closed-form expressions for the moment generating function (MGF) of the filtered output of square envelope receivers with signal and colored Gaussian noise input are derived. The informative signal is a binary sequence of rectangular pulses. The considered Gaussian processes are the Wiener process, a Gaussian process with linear covariance (moving average), and the Ornstein-Uhlenbeck process. The derived MGFs are then applied to the problem of finding the quantum limit for optically preamplified, direct detection receivers.
\end{abstract}

Index Terms-Communication theory, envelope receivers, error analysis, optical communications, preamplified receivers.

\section{INTRODUCTION}

D ETERMINING the distribution of the output of square envelope receivers with colored Gaussian input constitutes a classic problem in communication theory; see, e.g., [1]-[5]. Although the mathematical formalism for determining the statistics of the output of such receivers is well known, e.g., [1], [3], [5], and [6], deriving closed-form expressions for the distribution is a complex task. Moreover, if both signal and noise are present at the input, this task become even more formidable. For the case of noise only input expressions for the moment generating function (MGF) and analytical approximations to the probability density function have been reported for several covariance kernels, e.g., [3], [5], and [7]. For both signal and noise being present at the input closed-form expressions for the distribution are scarcely documented in the literature. In this letter, closed-form expressions for the MGF are derived for the case of an input informative signal composed of a binary sequence of rectangular pulses. The considered Gaussian processes are the Wiener process, a Gaussian process with linear covariance (moving average), and the Ornstein-Uhlenbeck process.

The remain of this letter is structured as follows. In Section II the system model to be discussed is presented. The mathematical formalism to obtain closed-form expressions for the MGF of the receiver's output is also described. Closed-form expressions for the MGF for the considered Gaussian processes are derived in Section III. Section IV is devoted to the application of the derived MGFs to find the quantum limit for optically preamplified, direct detection receivers. Finally, summarizing conclusions are presented in Section V.

Paper approved by O. K. Tonguz, the Editor for Optical Transmission Systems of the IEEE Communications Society. Manuscript received August 15, 1998; revised December 15, 1999.

The author is with Eindhoven University of Technology, Telecommunications Technology, and Electromagnetics, $5600 \mathrm{MB}$ Eindhoven, The Netherlands (e-mail: i.tafur@tue.nl).

Publisher Item Identifier S 0090-6778(01)00262-8.

\section{SYSTEM MODEL}

In this section, we present the reference model for the system under investigation. The receiver schematic diagram is presented in Fig. 1. This receiver has the classic configuration of pre-detection filter $r(t)$, square envelope detector, and post-detection filter. The input is an informative signal corrupted by an additive white Gaussian noise (AWGN) $n(t)$ with spectral density parameter $N_{0}$. In the sequel, equivalent bandpass representation of signal and noise is assumed. The incoming signal is a binary sequence of rectangular pulses $S(t)$. For a given bit pattern, $\mathcal{B}=\left(\ldots, b_{-1}, b_{o}, b_{1}, \ldots\right)$, at the output of the filter $r(t)$ the signal is given by

$$
\begin{aligned}
Y(t) & =S(t) \star r(t) r \\
& =\sqrt{\frac{m}{T}}\left[b_{0} l(t)+\sum_{k \neq 0} b_{k} l(t-k T)\right]
\end{aligned}
$$

where $r(t)$ is the pre-detection filter impulse response, $l(t)=$ $g(t) \star r(t)$ in which $\star$ denotes convolution, $g(t)$ is unit rectangular pulse of duration $T, b_{k} \in\{0,1\}$ are statistically binary symbols representing a data "zero" and a "one," respectively. $m$ is the energy content of the signal $S(t)$ in a bit-duration time interval $m=(1 / 2) \int_{0}^{T}|S(t)|^{2} d t$. At the pre-detection filter output, the resultant colored Gaussian noise is denoted by $X(t)$, whose in-phase and quadrature components have zero mean and autocovariance

$$
K(\tau)=\frac{N_{0}}{2} R(\tau) \quad R(\tau)=\int_{-\infty}^{\infty} r(t) r^{*}(t+\tau) d t
$$

where $*$ means complex conjugate. With the above notations, the input of the square envelope detector becomes $B(t)=Y(t)+X(t)$. If we consider and integrate-and-dump post-detection filter, then the receiver's output is given by

$$
\Lambda=\int_{0}^{T}|Y(t)+X(t)|^{2} d t .
$$

The general mathematical form for the MGF of $\Lambda, M_{\Lambda}(s)=$ $\mathrm{E}\left\{e^{s \Lambda}\right\}$, is well known, e.g., [1], [7]

$$
M_{\Lambda}(s)=\prod_{n=1}^{\infty} \frac{1}{\left(1-\lambda_{n} s\right)} \exp \left(\sum_{n=1}^{\infty} \frac{\left|y_{n}\right|^{2} s}{1-\lambda_{n} s}\right)
$$

where $y_{n}=\int_{0}^{T} Y(t) f_{n}^{*}(t) d t$. The set of orthonormal functions $\left\{f_{n}\right\}$ are the eigenfunctions and $\lambda_{n}$ are the eigenvalues of the integral equation

$$
\int_{0}^{T} K(t, u) f_{n}(u) d u=\lambda_{n} f_{n}(t), \quad 0 \leq t \leq T
$$




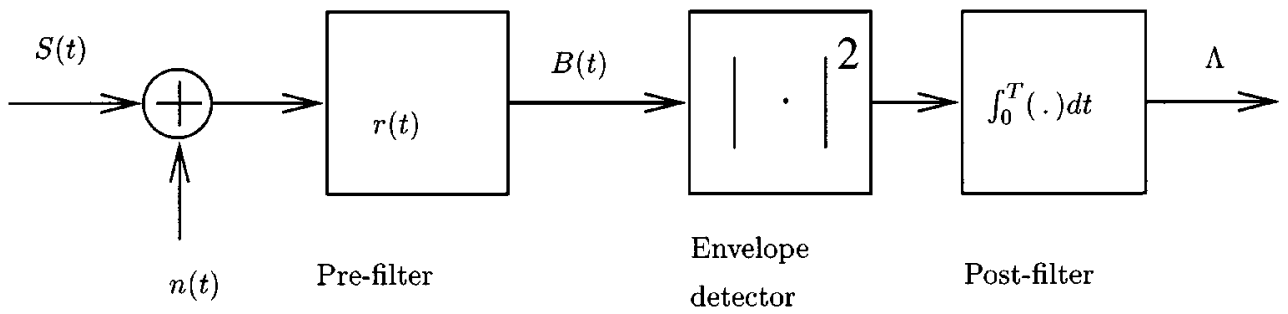

Fig. 1. Square envelope receiver.

in which $K(t, u)$ is the covariance kernel of the process $X(t)$. Let $h(t, u ; s ; \tau)$ stand for the resolvent kernel associated with the integral equation (5). The MGF in (4) can be represented in terms of the resolvent kernel as [7]

$$
M_{\Lambda}(s)=[D(s)]^{-1} \exp [F(s)]
$$

where

$$
\begin{aligned}
F(s)= & s \int_{0}^{T}|Y(t)|^{2} d t \\
& +s^{2} \int_{0}^{T} \int_{0}^{T} Y^{*}(t) h(t, u ; s ; T) Y(u) d t d u
\end{aligned}
$$

and $D(s)$, also called the Fredholm determinant, is given by

$$
D(s)=\exp \left[\int_{0}^{s} \int_{0}^{T} h(t, t ; v ; T) d t d v\right] .
$$

\section{MGF}

In this section, closed-form expressions for $M_{\Lambda}(s)$ are presented for three different Gaussian processes.

Case 1: The Wiener Process: Suppose that $r(t)$ is the (normalized) impulse response of an integrate-and-dump filter, then

$$
\begin{aligned}
X(t) & =\int_{0}^{\infty} r(t-s) n(s) d s \\
r(t-s) & = \begin{cases}\frac{1}{T}, & s<t \\
0, & s>t .\end{cases}
\end{aligned}
$$

The process $X(t)$ is the Wiener process which has covariance given by

$$
K(t, u)=\frac{N_{0}}{T^{2}} \int_{0}^{t} \int_{0}^{u} \delta(t-u) d t d u=\frac{N_{0}}{T^{2}} \min (t, u) .
$$

As one can observe from the character of $r(t)$, the integrate-and-dump filter does not introduce intersymbol interference (ISI). The resulting expression for the MGF is (see Appendix A for a derivation)

$$
M_{Z}(s)=\cos \left(\sqrt{N_{0} s}\right)^{-1} \exp \left[-\frac{m}{N_{0}}+\frac{m}{N_{0}} \frac{\tan \left(\sqrt{N_{0} s}\right)}{\sqrt{N_{0} s}}\right] \text {. }
$$

Case 2: Gaussian Process with Linear Covariance: Let $r(t)$ be a finite-time bandpass integrator (moving average) whose impulse response is given by

$$
r(t)= \begin{cases}\frac{1}{T}, & 0 \leq t \leq T \\ 0, & \text { otherwise }\end{cases}
$$

The covariance kernel of $X(t)$ is the triangular function (linear covariance [5])

$$
K(\tau)= \begin{cases}\frac{N_{0}}{T}\left(1-\frac{|\tau|}{T}\right), & 0 \leq t \leq T \\ 0, & \text { otherwise }\end{cases}
$$

The analysis for the observation time $[0, T]$ shows that the MGF for $\Lambda$ is of the same character as (11). As already reported in [5], it can be derived from (11) by substitution of $\sqrt{s}$ by $\sqrt{2 s}$. The result is

$$
M_{Z}(s)=\cos \left(\sqrt{2 N_{0} s}\right)^{-1} \exp \left[-\frac{m}{N_{0}}+\frac{m}{N_{0}} \frac{\tan \left(\sqrt{2 N_{0} s}\right)}{\sqrt{2 N_{0} s}}\right] .
$$

In contradistinction to the integrate-and-dump filter the finite-duration integrator introduces ISI. Communication is only possible if the observation time is shifted from $[0, T]$ to $[(T / 2),(3 T / 2)]$. For this type of filter, only a single past and one succeeding bit produce ISI on the present transmitted bit. Hence the bit sequence of interest is $\mathcal{B}=\left(b_{-1}, b_{0}, b_{1}\right)$. The expression for $F(s)$ is given by (14), shown at the bottom of the page, with

$$
\begin{aligned}
H_{1}(s)= & \operatorname{sinc}(\beta)\left(5 N_{0} s+4\right)-2 \operatorname{sinc}(\beta / 2)\left(N_{0} s+1\right) \\
& -2 \cos (\beta / 2) \\
H_{2}(s)= & \operatorname{sinc}(\beta)\left(N_{0} s-4\right)-\operatorname{sinc}(\beta / 2)\left(N_{0} s-2\right) \\
& +2 \cos (\beta / 2) \\
H_{3}(s)= & \operatorname{sinc}(\beta)\left(N_{0} s / 2+2\right)-\operatorname{sinc}(\beta / 2)-\cos (\beta / 2)
\end{aligned}
$$

where $\beta=\sqrt{2 N_{0} s}$, and $\operatorname{sinc}(x)=\sin (x) / x$. The Fredholm determinant is given by $D(s)=\cos \left(\sqrt{2 N_{0} s}\right)$. Appendix B explains the derivation of (14).

Case 3: The Ornstein-Uhlenbeck Process: If $r(t)$ is the impulse response of the Lorentzian filter

$$
r(t)=\sqrt{2} \mu e^{-\mu t}, \quad t \geq 0
$$

$$
F(s)=\frac{m\left[b_{0} H_{1}(s)+\left(\sqrt{b_{-1} b_{0}}+\sqrt{b_{0} b_{1}}\right) H_{2}(s)+\left(b_{-1}+b_{1}\right) H_{3}(s)\right]}{4 N_{0} \cos (\beta)}
$$




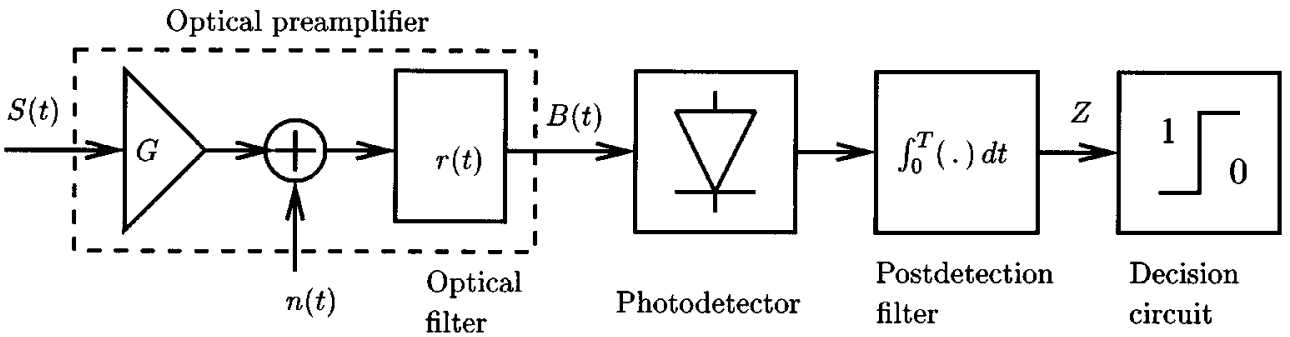

Fig. 2. Optically preamplified OOK receiver.

then the process $X(t)$ is the so called Ornstein-Uhlenbeck process with covariance given by

$$
K(\tau)=\mu e^{-\mu|\tau|}, \quad \tau \geq 0 .
$$

The information signal for $t \in[0, T]$ is given by

$$
\begin{aligned}
Y(t) & =\sqrt{\frac{2 m}{T}}\left[b_{o}+\rho e^{-\mu t}\right] \\
\rho & =\left(e^{\mu T}-1\right) \sum_{k=-\infty}^{-1} b_{k} e^{\mu k T}-b_{\circ} .
\end{aligned}
$$

The Lorentzian filter is a causal filter; hence when studying the effect of ISI only a sequence of previous bits with respect to the present transmitted bit $b_{0}$ is treated, $\mathcal{B}=\left(\ldots, b_{-2}, b_{-1}, b_{0}\right)$. In practice only a small number of previous bit is considered [8] For this case the resolvent kernel is well known, e.g., [5] and [7]. The closed-form expression for $M_{\Lambda}(s)$ and its detailed derivation has been reported in an earlier paper by the author [8]. The resulting expressions for $D(s)$ and $F(s)$ are presented here

$$
F(s)=\frac{2 m G}{\mu T}\left[b_{o}^{2} F_{1}(s)+b_{\circ} \rho F_{2}(s)+\rho^{2} F_{3}(s)\right]
$$

where

$$
\begin{aligned}
& F_{1}(s) \\
& \quad=\mu T s+\frac{2 \mu T s^{2} \sigma^{2}}{v^{2}} \\
& \quad+\frac{4 s^{2} \sigma^{2}\left(2-\left[(v+1) e^{\beta T}-(v-1) e^{-\beta T}\right]\right)}{v^{3}\left[(v+1)^{2} e^{\beta T}-(v-1)^{2} e^{-\beta T}\right]} \\
& F_{2}(s) \\
& =2 s\left[1-\frac{4-2 s \sigma^{2}\left[(v+2) e^{\beta T}-(v-2) e^{-\beta T}\right]}{v\left[(v+1)^{2} e^{\beta T}-(v-1)^{2} e^{-\beta T}\right]}\right] \\
& F_{3}(s) \\
& =s\left[\frac{1}{2}+\frac{\sigma^{2} s\left(e^{\beta T}-e^{-\beta T}\right)-\left[(v-1) e^{\beta T}+(v+1) e^{-\beta T}\right]}{\left[(v+1)^{2} e^{\beta T}-(v-1)^{2} e^{-\beta T}\right]}\right]
\end{aligned}
$$

in which $v=\sqrt{1-2 \sigma^{2}}, \beta=v \mu$.

$$
D(s)=\frac{(v+1)^{2} e^{\beta T}-(v-1)^{2} e^{-\beta T}}{4 v e^{\mu T}} .
$$

The mean and the variance of $Z$ can be found from the properties of the MGF. Namely, from the first and second derivative of the MGF evaluated at $s=0$ [9]. The mean and the variance of $Z$ can also be expressed in terms of the covariance kernel without the knowledge of the MGF [8]. The validity of the previous derived MGF has been tested by confirming that the mean and the variance obtained by both methods are identical. Moreover, if only noise is present, then the MGF is given only in terms of the Fredholm determinant $D(s)$, and their expressions are in agreement with those already known in the literature, e.g., [5] and [7].

\section{APPLiCATIONS}

In this section, we applied the derived MGF to determine the quantum limit for optically preamplified, on-off keying (OOK) direct detection receivers. The schematic diagram of such a receiver is illustrated in Fig. 2. The preamplifier is an EDFA (erbium-doped fiber amplifier) which is modeled as linear optical field amplifier with gain $G$ and AWGN noise $n(t)$ representing the ASE (amplified spontaneous emission) noise. The spectral parameter of $n(t)$ is given by $N_{0}=n_{\mathrm{sp}}(G-1) h \nu$, where $n_{\mathrm{sp}}$ is the amplifier spontaneous emission factor, $h$ is the Planck's constant, and $\nu$ optical frequency. An optical filter $r(t)$ is used to limit the effect of ASE on the system performance, and in the case of WDM (wavelength-division multiplexing) systems to select the desired channel.

By introducing a proper normalization (see [8]), $m$ represents the average number of photons contained in an optical signal $S(t)$ for a transmitted binary "one." The spectral parameter of $n(t)$ is then given by $N_{0}=n_{\mathrm{sp}}(G-1)$. At the output of the photodetector the photocurrent is directly proportional to the square magnitude of the received optical field. Further, the photocurrent is filtered and sampled to form the decision variable $Z$. Thus, the analysis of optically preamplifed, OOK direct detection receivers (Fig. 2) is an example of the classic communication situation of square envelope detectors followed by filtering with colored Gaussian input. Assume that the postdetection filter is an integrate-and-dump filter. The MGF for the receiver decision variable $Z$ is then given by $M_{Z}(s)=M_{\Lambda}\left(e^{s}-\right.$ 1 ), where $\Lambda$ is the so-called Poisson parameter [cf. (3)], e.g., [10] and [8].

Assuming independent, equally likely binary symbols the average error probability is given by

$$
P_{e}=\frac{1}{2}\left[\mathrm{E}_{\mathcal{B}}\left\{P_{r}\left(Z<\left.\alpha\right|_{\mathcal{B} \mid b_{0}=1}\right)+P_{r}\left(Z>\left.\alpha\right|_{\mathcal{B} \mid b_{0}=0}\right)\right\}\right] .
$$

Based on the MGF for the decision variable $Z$, error probabilities are expeditiously computed by the so-called saddlepoint approximation. For further details on the saddlepoint approximation see [7], [11], and [8] for an application to performance analysis of optically preamplified receivers.

In optical communications, the (standard), quantum limit is defined as the average number of photons per bit in the optical signal $S(t)$ needed to achieve a bit-error probability of $10^{-9}$ 
TABLE I

QUANTUM LIMIT FOR OPTICALLY PREAMPLIFIED OOK DiRECT DETECTION, $G=100$, AND $n_{\mathrm{sp}}=1$

\begin{tabular}{l|c}
\hline Optical filter type & Quantum limit \\
\hline \hline Ideal bandpass & $38.4[11]$ \\
\hline Integrate-and-dump & 46.3 \\
\hline Finite-time Bandpass Integrator & 59.6 \\
\hline Lorentzian, Optimal $\mu T=7$ & 49.9 \\
\hline
\end{tabular}

assuming ideal detection conditions, which for a preamplified receiver means that a large $G$ is assumed.

Suppose we have optical filters described by the impulse responses (equivalent baseband representation) of Cases 1-3 [see (9), (12), and (15)]. The above derived MGFs [(11), (14), and (17)] can then be used [substituting $m \mapsto m G$ to account for amplification, and with $\left.N_{0}=n_{\mathrm{sp}}(G-1)\right]$ to find the corresponding quantum limits. In Table I, the obtained results are presented. For comparison, the quantum limit for the situation when the optical signal is assumed to pass the optical filter undistorted and that $X(t)$ is Gaussian bandlimited (ideal bandpass filter) is also included. When the effect of optical filtering is taken into account penalties are observed compared to the case assuming ideal bandpass filtering. For the analyzed optical filters, this penalty in the quantum limit is at least of eight photons per bit. It should be noted that of the considered optical filters only the Lorentzian filter (Case 3) represents practical interest. Widely used in optical transmission systems Fabry-Perot filters are well described by the Lorentzian impulse response of (15).We observe also that there exists an optimum bandwidth bit-time product $B T=\mu T / \pi$, the reason being a tradeoff between ISI and ASE noise (see Table I).

\section{Conclusions}

Closed-form expressions, believed to be new, for the MGF of the filtered output of square envelope receivers with signal and colored Gaussian noise have been derived. The Wiener process, a Gaussian process with linear covariance, and the Ornstein-Uhlenbeck process are considered. The informative signal is binary sequence of rectangular pulses. We present an application of the derived MGFs in the performance analysis of optically preamplified, direct detection receivers.

APPENDIX A

DERIVATION OF THE MGF: CASE 1

For the covariance function given by (10), the resolvent kernel is given by [5]

$$
\begin{aligned}
& h(t, u ; s ; \tau) \\
& =\underbrace{\frac{\sigma^{2}}{\beta}[\sin (\beta t) \cos (\beta u)-\theta(t-u) \sin (\beta t) \cos (\beta u)]}_{h_{1}} \\
& +\underbrace{q \frac{\sigma^{2}}{\beta} \sin (\beta t) \sin (\beta u)}_{h_{2}}+\underbrace{\theta(t-u) \frac{\sigma^{2}}{\beta} \cos (\beta t) \sin (\beta u)}_{h_{3}}
\end{aligned}
$$

where $\sigma^{2}=N_{0} / T^{2}, \beta=\sqrt{\sigma^{2} s}$

$$
q=\tan (\beta T) \quad \text { and } \quad \theta(t-u)= \begin{cases}0, & t<u \\ 1, & t>u .\end{cases}
$$

We perform integration in (7) first with respect to $t$ and with respect to $u$.

$$
\begin{aligned}
& I_{1}=\int_{0}^{T} Y^{*}(u) \underbrace{\int_{0}^{T} h(t, u ; s ; T) Y(t) d t}_{I_{2}} d u \\
& I_{2}=\int_{0}^{T} \underbrace{Y(t) h_{1}}_{a}+\underbrace{Y(t) h_{2}}_{b}+\underbrace{Y(t) h_{3}}_{c} d t
\end{aligned}
$$

in which $Y(t)=\sqrt{(2 m / T)} t / T \equiv A t$. Solving the integrals $a, b, c$, we get

$$
I_{2}=-A \frac{\sigma^{2} u}{\beta^{2}}+A \frac{\sigma^{2}}{\beta^{3}} \frac{\sin (\beta u)}{\cos (\beta T)} .
$$

Subsequently

$$
\begin{aligned}
I_{1} & =\int_{0}^{T} A u I_{2} d u=-A^{2} T^{3} \frac{\sigma^{2}}{3 \beta^{2}}+\frac{A^{2} \sigma^{2}}{\beta^{5}}(\tan (\beta T)-\beta T) . \\
F(s) & =s \int_{0}^{T}|Y(t)|^{2} d+s^{2} I_{1} \quad \text { resulting in } \\
F(s) & =-\frac{m}{N_{0}}+\frac{m}{N_{0}} \frac{\tan \left(\sqrt{N_{0} s}\right)}{\sqrt{N_{0} s}} .
\end{aligned}
$$

The Fredholm determinant is given by [see (8)]

$$
D(s)=\exp \left\{\int_{0}^{s} \frac{\sigma T}{\sqrt{v} 2} \tan (\sigma T \sqrt{v}\} d v=\cos \left(\sqrt{N_{0}} s\right) .\right.
$$

A result already obtained in [5] and references therein.

\section{APPENDIX B \\ DERIVATION OF THE MGF: CASE 2}

For the covariance kernel given in (13) and an observation interval $[-T / 2, T / 2]$, the resolvent kernel is presented in [5]. If the observation time is extended to $[0,2 T]$, then the resultant resolvent kernels is given by

$$
\begin{aligned}
& h(t, u ; s ; T) \\
& \quad=\frac{N_{0}}{\beta T}\left[\tan \beta \cos \left[\frac{\beta(t-u)}{T}\right]-\sin \left[\frac{\beta|t-u|}{T}\right]\right]
\end{aligned}
$$

with $\beta=\sqrt{2 N_{0} s}$. The MGF is found by performing integration in (7) and (8) with the proper integration limits and the corresponding expression for $Y(t)$. The algebraic procedure is similar to that presented in Appendix A.

\section{REFERENCES}

[1] M. Kac and A. J. F. Siegert, "On the theory of noise in radio receivers with square law detectors," J. Appl. Phys., vol. 18, pp. 383-397, Apr. 1947.

[2] S. O. Rice, "Mathematical analysis of random noise," Bell Syst. Tech. J., vol. 23 and 24, pp. 282-332-46-156, 1954.

[3] A. J. F. Siegert, "A systematic approach to a class of problems in the theory of noise and others random phenomena-Part II: Examples," IEEE Trans. Inform. Theory, vol. IT-3, pp. 38-43, Mar. 1957. 
[4] D. Slepian, "Fluctuations of random noise power," Bell Syst. Tech. J. pp. 163-184, Jan. 1958.

[5] M. I. Schwartz, "Distribution of the time-average power of a Gaussian process," IEEE Trans. Inform. Theory, vol. IT-16, pp. 17-26, Jan. 1970.

[6] C. W. Helstrom, "Distribution of the filtered output of a quadratic rectifier computed by numerical contour integration," IEEE Trans. Inform. Theory, vol. IT-32, pp. 450-463, July 1986.

[7] - Elements of Signal Detection and Estimation. Englewood Cliffs, NJ: Prentice-Hall, 1995.

[8] I. T. Monroy and G. Einarsson, "Bit error evaluation of optically preamplified direct detection receivers with Fabry-Perot optical filters," $J$. Lightwave Technol., vol. 15, pp. 1546-1553, Aug. 1997.
[9] A. Papoulis, Probability, Random Variables, and Stochastic Processes, 2nd ed. New York: McGraw-Hill, 1991.

[10] S. D. Personick, "Applications for quantum amplifiers in simple digital optical communication systems," Bell Syst. Tech. J., vol. 52, pp. 117-133, Jan. 1973.

[11] G. Einarsson, Principles of Lightwave Communications. New York: Wiley, 1996. 